\title{
Degradación y durabilidad del acero en el hormigón: Evaluación por
}

\section{técnicas electroquímicas}

\author{
J.A. González*, J.M. Miranda** y S. Feliu*
}

\begin{abstract}
Resumen Se analiza someramente la importancia técnica y económica de las estructuras de hormigón armado (EHA), los factores de corrosión que pueden limitar su durabilidad, así como las formas de evaluar la cinética de deterioro mediante técnicas electroquímicas, en el laboratorio y directamente, in-situ sobre las estructuras reales. Se resalta la labor desarrollada en el CENIM, en colaboración con el ICCET, desde el decenio de los 70, desarrollando técnicas electroquímicas de estudio de la corrosión aplicables al sistema acero/hormigón, de enorme sensibilidad en comparación con las técnicas precedentes, lo que ha permitido dar un contenido cuantitativo a multitud de conocimientos cualitativos adquiridos hasta entonces.
\end{abstract}

Palabras clave Acero. Corrosión. Hormigón. Técnicas electroquímicas. Medidas en campo.

\section{Degradation and durability of steel in concrete: Evaluation by electrochemical techniques}

\begin{abstract}
A brief analysis is made of the technical and economic importance of reinforced concrete structures, the corrosion factors that can limit their durability, and the ways of evaluating deterioration kinetics using electrochemical techniques in the laboratory and in-situ on real structures. Attention is drawn to the work performed at CENIM since the 1970's, in collaboration with the ICCET, developing electrochemical techniques for studying corrosion that are applicable to the steel/concrete system. These techniques are enormously sensitive compared to those previously available and have allowed a quantitative content to be given to much of the qualitative knowledge acquired hitherto.
\end{abstract}

Keywords Steel. Corrosion. Concrete. Electrochemical techniques. Field measurements.

\section{INTRODUCCIÓN}

Se estima que el $30 \%$ de la producción mundial de acero se destina a la reposición de las pérdidas causadas por la corrosión. Admítase cierto error en la estimación, pero resulta incontestable la enorme repercusión de los costes directos de la corrosión. Pues bien, un $18 \%$ de los costes se atribuyen al sector de la construcción ${ }^{[1]}$, uno de los que más influyen en el empleo y el nivel de vida. Dentro de este sector, la construcción de estructuras de hormigón, en masa y armado, supera en tonelaje a las de todos los otros materiales considerados conjuntamente, siendo responsable de la mayoría de los costes mencionados. Concretamente, en España se atribuyen a la corrosión en el sector de la cons- trucción, pérdidas anuales por valor de $1.200 \mathrm{mi}$ llones de euros ${ }^{[2]}$.

Afortunadamente, el elevado $\mathrm{pH}$ del hormigón garantiza, si se procede correctamente, el estado pasivo de las armaduras. En el estado pasivo la corrosión es insignificante y, si se mantiene, la durabilidad de las EHA es prácticamente ilimitada.

Sin embargo, en unos cuantos casos especiales, que siempre se presentan ligados a la pérdida del estado pasivo, la corrosión de los refuerzos se hace significativa, provocando, a veces, una dramática reducción en la durabilidad de las EHA. Los factores desencadenantes de la corrosión son, la inmensa mayoría de las veces, la carbonatación del hormigón o la penetración de cloruros hasta el nivel de las armaduras por encima de un cierto umbral

$\left(^{*}\right) \quad$ Centro Nacional de Investigaciones Metalúrgicas, Avda. Gregorio del Amo, 8. 28040, Madrid.

${ }^{* *}$ Instituto de Metalurgia de la Universidad Autónoma de San Luis Potosí, Avda. Sierra Leona, 550. San Luis Potosí, México. 
de concentraciones, fijado en un $0,4 \%$ con relación al peso de cemento en la EHE ${ }^{[3]}$.

La predicción de la vida residual en servicio de las estructuras dañadas por corrosión, el control de la eficacia de las medidas protectoras adoptadas, de las técnicas de rehabilitación aplicadas o de las reparaciones efectuadas requieren, necesariamente, un procedimiento de medida cuantitativa de la velocidad de corrosión de los refuerzos. En la presente comunicación se analizan las dificultades para lograr este objetivo en el laboratorio e in-situ, describiendo someramente la labor desarrollada por el CENIM con las técnicas electroquímicas de estudio de la corrosión, las que ofrecen las mayores posibilidades al respecto.

\section{MATERIALES Y PROCEDIMIENTO EXPERIMEN- TAL}

No se han realizado ensayos especiales para este trabajo. Se ha aprovechado la experiencia acumulada de muchos años en esta temática. La mayoría de los resultados a los que se hace referencia se han obtenido en el laboratorio con pequeñas probetas de mortero, muy manejables, con redondos de acero para armar embebidos en ellas para estudiar su comportamiento. Este tipo de muestras permite analizar el efecto de los factores de corrosión dependientes del propio mortero u hormigón, de la armadura y del medio ambiente ${ }^{[4]}$.

Se han uțilizạado también vigas y losas de dimensiones considerables (Fig. 1) para tratar de analizar la respuesta de los métodos electroquímicos de estimación de la velocidad de corrosión en

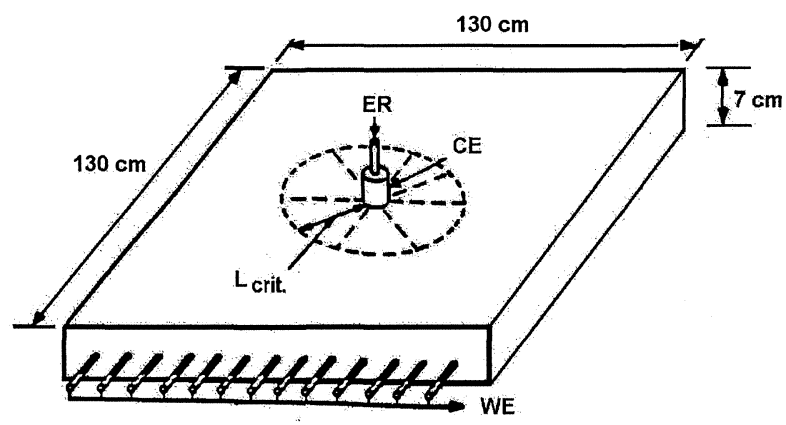

Figura 1. Losa de mortero de cemento reforzada con 13 armaduras de acero, mostrando la disposición de los electrodos para realizar las medidas electroquímicas y la longitud crítica hasta la que se extienden las líneas de corriente.

Figure 1. Cement mortar slab reinforced with 13 steel rebars, with the electrode arrangement to apply the electrical signal and the idealized critical length for current lines. condiciones semejantes a las estructuras reales $\left.{ }^{[5} \mathrm{y} 6\right]$. En una etapa posterior, se han utilizado los mismos procedimientos in-situ ${ }^{[7}$ y 8$]$.

\section{RESULTADOS}

Quiere resaltarse que:

- Las medidas in-situ, sobre las estructuras reales, se llevan a cabo imponiendo señales eléctricas con pequeños contraelectrodos al conjunto de armaduras que, normalmente, están todas en cortocircuito. La consecuencia es que las líneas de corriente se dispersan a partir del pequeño contraelectrodo emisor, atenuándose progresivamente hasta extinguirse a una longitud crítica desconocida en cada caso (Fig. 1). Se desconoce el área que interviene en las medidas y no puede calcularse una velocidad de corrosión por la clásica fórmula de Stern y Geary ${ }^{[9]}$, que presupone un reparto uniforme de la corriente fácil de conseguir en el laboratorio. Sirvan como ejemplo los datos de la figura 2, que muestran la atenuación de la polarización aplicada con la distancia, en el caso de dos losas como la de la figura 1 , una pasiva y otra con adiciones de cloruros (activa).

- Si no se tiene en cuenta la dispersión de las señales eléctricas pueden cometerse errores enormes en la estimación de las velocidades de corrosión, como queda patente en la figura 3,

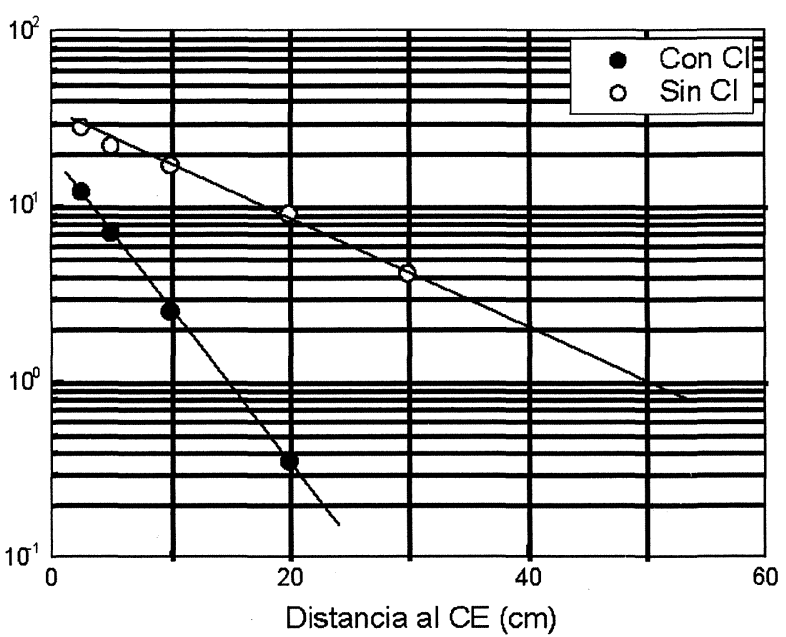

Figura 2. Atenuación de la polarización aplicada con la distancia al pequeño CE emisor. Comportamientos típicos en armaduras activas $(\bullet)$ y pasivas (o).

Figure 2. Attenuation of the applied polarization with the distance from the small CE. Typical behaviours of active ( $\bullet$ ) and passive (o) reinforcements.

Rev. Metal. Madrid Vol. Extr. (2005) 279-283 


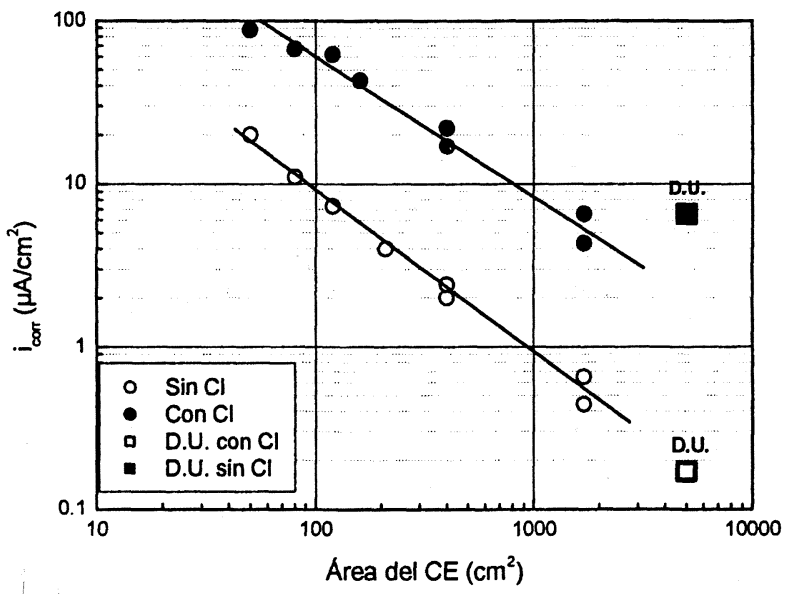

Figura 3. Valores estimados para la $i_{\text {corr }}$ en losas con $(\bullet)$ y $\sin (0)$ adiciones de cloruros en función del tamaño del CE empleado.

Figure 3. Stimates values of $i_{\text {corr }}$ in concrete slabs with (•) and without (o) chloride additions versus size of the CE.

donde se representan las velocidades estimadas a partir de la resistencia de polarización aparente $\left(R_{\text {pap }}\right)$ para medidas efectuadas con contraelectrodos de tamaño diferente. Las medidas correctas de $i_{\text {corr }}$ se obtienen al forzar una distribución uniforme de las líneas de corriente (Puntos D.U. en la figura).

\section{DISCUSIÓN}

\subsection{Unas palabras sobre el estado del arte}

Hasta los años 70, en los que se inició una larga colaboración del CENIM y el ICCET ${ }^{[10 \text { y }}{ }^{11]}$, la investigación sobre la corrosión en el hormigón armado resultaba tremendamente dificultada por la falta de acceso directo a las armaduras y por la necesidad de romper las muestras para obtener un sólo dato, pues la evaluación se realizaba exclusivamente por procedimientos visuales o gravimétricos.

Con el desarrollo de las técnicas electroquímicas de estudio de la corrosión, a partir del decenio de los $60^{[9]}$, se superaron estas dificultades y los avances fueron mucho más rápidos, al disponer de métodos cuantitativos y no destructivos de evaluación de la velocidad de corrosión que, con una sola muestra, permitían seguir la evolución del proceso de corrosión a lo largo del tiempo.

La labor conjunta CENIM-ICCET, permitió un avance mucho más rápido en los estudios de la corrosión de las EHA y dar forma cuantitativa a muchos de los conocimientos cualitativos ya conocidos. La forma general de proceder es imponer al sistema estudiado una señal eléctrica y, del análisis de la respuesta, obtener la información deseada. La técnica que se desarrolló más rápidamente consiste en determinar la "resistencia de polarización" del sistema, dada por la relación entre la señal que le es impuesta (polarización), generalmente de unos pocos $\mathrm{mV}$ y la respuesta en intensidad, $R_{p}=$ $\Delta \mathrm{E} / \Delta \mathrm{I}$. Una vez determinado el valor de $\mathrm{R}_{\mathrm{p}}$, es inmediato el cálculo de la velocidad instantánea de corrosión a partir de la conocida fórmula de Stern ${ }^{[9]}$.

La aplicación de estas técnicas en el laboratorio no ofrece dificultad alguna. Puede comprobarse así, que las $i_{\text {corr }}$ de las armaduras activas pueden llegar a ser unas 100 veces superiores a las propias del estado pasivo ${ }^{[4-8]}$. Es decir, las durabilidades residuales previsibles de las EHA que se corroen en el estado activo pueden reducirse por un factor similar. Sin embargo, las dificultades para llegar a estimaciones seguras de las $i_{\text {corr }}$ se multiplican en las medidas in-situ, sobre estructuras reales.

\subsection{Medidas in-situ}

La fuente principal de inexactitudes reside, como ya se ha comentado, en que las señales eléctricas se extienden, a partir del contraelectrodo utilizado para imponerlas, una longitud crítica desconocida (Fig. 1), sin que la $R_{p}$ aparente estimada pueda atribuirse a un área determinada, no pudiendo calcularse la velocidad de corrosión a partir de la fórmula de Stern.

La posibilidad de recurrir a contraelectrodos del mismo tamaño que las estructuras para conseguir, como en el laboratorio, un reparto uniforme de las señales eléctricas, resulta totalmente impracticable. La consecuencia es que no existían, hasta fechas muy recientes, técnicas no destructivas que permitieran obtener, de modo fiable, información sobre las velocidades de corrosión en grandes estructuras $^{[5-8]}$. El problema se puede afrontar de varios modos, en el CENIM, en colaboración con el ICCET, se ha investigado en varias direcciones:

- A través de un circuito equivalente (CE) en "línea de transmisión" (Fig. 4), que reproduce aproximadamente el sistema y ofrece la posibilidad de un tratamiento matemático que tiene en cuenta la distribución heterogénea de las señales eléctricas ${ }^{[5}$ y 6$]$.

- Forzando una distribución uniforme de las líneas de corriente en una pequeña zona de superficie conocida, confinándolas por medio de 


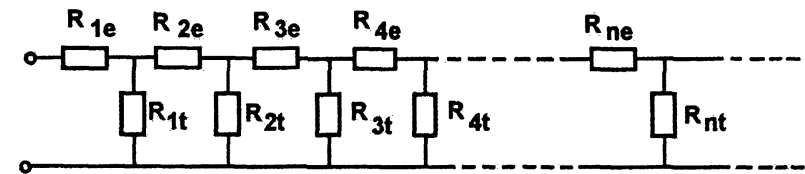

Figura 4. Modelo de CE en línea de transmisión para simular la respuesta de los refuerzos al aplicarles señales eléctricas de pequeña amplitud.

Figure 4. Transmission line model for simulating the response of the reinforcements to small-amplitude electrical signals.

contraelectrodos auxiliares, como el anillo de guarda (Fig. 5) ${ }^{[7]}$.

- A partir de la atenuación de las señales eléctricas con la distancia ${ }^{[8]}$, mucho más rápida en las estructuras activas que en las pasivas (Fig. 2).

Este comportamiento es fácil de comprender a partir del CE de la figura 4, pues la resistencia de cada elemento de hormigón $\left(R_{n e}\right)$ no se diferencia mucho de unas estructuras a otras, mientras que la de cada elemento de armadura $\left(\mathrm{R}_{\mathrm{nt}}\right)$ crece, enormemente, en las pasivas, impidiendo que el acero actúe como sumidero de corriente, extendiéndose ésta lateralmente. La superficie afectada por la medida es, pues, mucho mayor en las estructuras pasivas, que pueden confundirse con las activas, cometiendo errores considerables en las estimaciones (Fig. 3) si no se confinan las líneas de corriente con el anillo de guarda (Fig. 5).

- Aplicando pulsos galvanostáticos de corta duración y analizando matemáticamente la atenuación (en el tiempo) de la polarización del electrodo a raíz de la interrupción de la corriente, mucho más rápida en las estructuras activas que en las pasivas ${ }^{[12}$ y 13$]$.

El procedimiento permite la determinación de la constante de tiempo del proceso de corrosión y, a partir de ella, de la velocidad de corrosión sin necesidad de conocer el área de las armaduras afectada por las medidas ${ }^{[12}$ y 13$]$

Los métodos explicados en los puntos 2, 3 y 4 han hecho posible el desarrollo de otros tantos prototipos de corrosímetros portátiles, uno de ellos, utilizado industrialmente desde hace años ${ }^{[7]}$. Se ha puesto, pues, en manos de técnicos e investigadores, una herramienta que facilita mucho la inspección en campo de las EHA.

\section{CONCLUSIONES}

- La colaboración del CENIM con el ICCET, desde el decenio de los 70, ha desarrollado técnicas electroquímicas de estudio de la corrosión aplicables al sistema acero/hormigón, de enorme sensibilidad en comparación con las técnicas precedentes, que han permitido dar un contenido cuantitativo a multitud de conocimientos cualitativos adquiridos hasta entonces.

- La dispersión de las señales eléctricas en las estructuras reales, hasta una longitud crítica desconocida en cada caso, impedía hasta épocas recientes conocer el área que intervenía en las medidas y estimar velocidades de corrosión fiables.
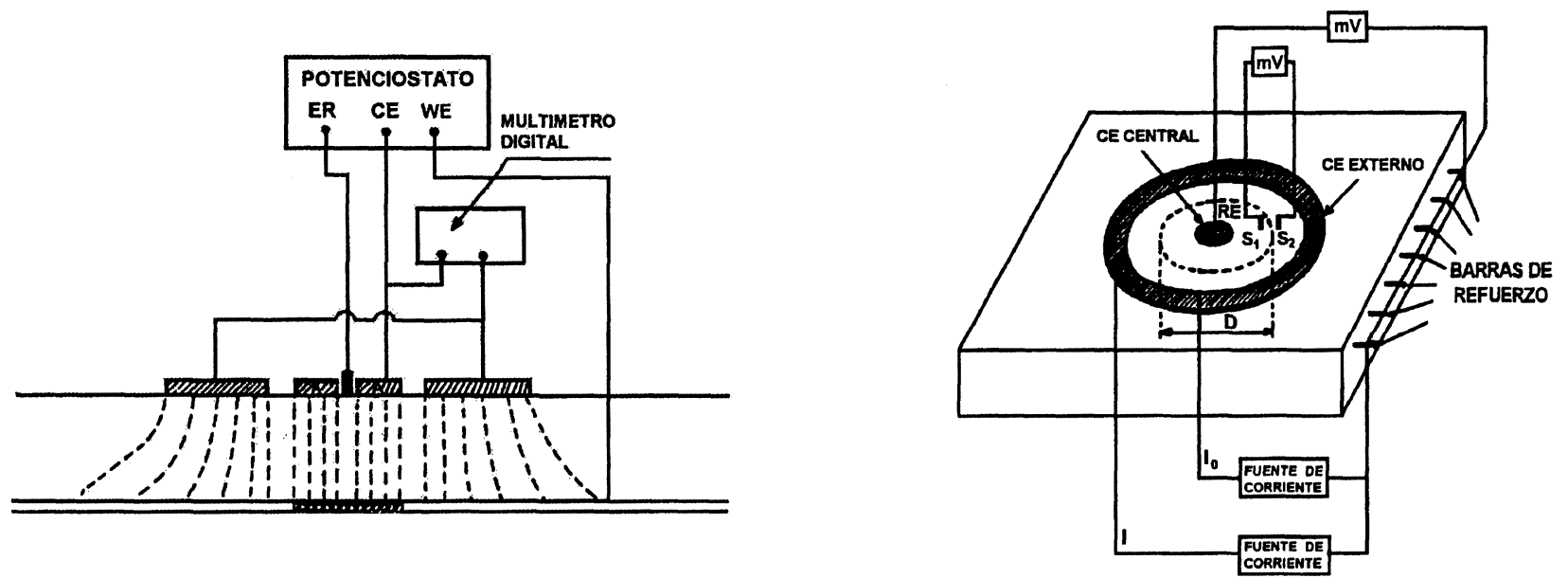

Figura 5. Un segundo contraelectrodo externo, concéntrico con el principal, conocido como "anillo de guarda", es la forma más eficaz de confinar las líneas de corriente en un área conocida.

Figure 5. A second external circular CE ("guard ring") concentric with the central CE, is the more efficient procedure for confining current lines from the central CE to within a specific area. 
- La colaboración CENIM-ICCET-GEOCISA ha cristalizado en el desarrollo de 3 prototipos de corrosímetros aplicables in-situ, basados, respectivamente, en el confinamiento de las líneas de corriente con un anillo de guarda, en la atenuación de las señales eléctricas con la distancia al pequeño electrodo emisor y, en la cinética de carga y descarga de las armaduras al aplicarles pulsos galvanostáticos de corta duración. El primero de tales corrosímetros ha sido patentado y se utiliza industrialmente desde hace, aproximadamente, un decenio.

\section{REFERENCIAS}

[1] Rep. Comm. Corros. Prot. (presidido por T.P. Hoar). Dept. of Trade and Industry. HMSO, Londres, 1971.

[2] R. MARTíneZ, Hormigón 38 (1998) 48-50.

[3] Ministerio de Fomento, Comisión Permanente del Hormigón, Instrucción del Hormigón Estructural EHE-98.

[4] S. Feliu, J.A. González, C. Andrade y V. Feliú, Corros. Sci. 29 (1989) 105-113.
[5] S. Feliu, J.A. González, C. Andrade y V. Feliú, Mater. Constr. 22 (1989) 199-205.

[6] J.A. González, S. Feliu, C. Andrade e I. RodríGuez, Mater. Constr. 24 (1991) 346-350.

[7] S. Feliú, J.A. González, V. Feliú, S. Feliú Jr., M.L. Escudero, J.A. Rodríguez-Maribona, V. Ausín, M.C. ANDRADE, J.A. Bolaño y F. JimÉnEZ, USA Patent 5, 259,944, 1993.

[8] S. Feliu, J.A. GonzÁlez y C. Andrade, J. Appl. Electrochem. 26 (1996) 305-309.

[9] M. Stern y A.L. Geary, J. Electrochem. Soc. 104 (1957) 56-63.

[10] C. Andrade y J.A. González, Ult. Avances Mater. Const. 150-151 (1974) 191-201.

[11] C. Andrade y J.A GonzÁlez, Werks. Korros. 29 (1978) 515-519.

[12] J.A. González, A. Cobo, M.N. GonzÁlez y S. Feliú, Corros. Sci. 43 (2001) 611-625.

[13] V. Feliu, A. Cobo, J.A. González y S. Feliú, Corros. 58 (2002) $72-81$. 